\title{
Metodología para la virtualización computacional de espacios físicos Universidad EAN
}

Fecha de recepción: 28 de febrero 2014

Fecha de aprobación: 05 de marzo 2014

Pp. $25-41$

\section{RESUMEN}

Este artículo presenta una metodología enfocada a proyectos de virtualización de espacios físicos. Con virtualización se hace referencia a proyectos en los que se digitaliza de manera interactiva un lugar físico. La metodología se presenta de manera detallada, así como algunos problemas que generalmente se presentan junto con sus posibles soluciones.

\section{Palabras Clave}

Virtualización, interactividad, modelado, texturizado y animación 3D. 


\section{Methodology for Physical Spaces \\ Computational virtualization}

\section{Abstract}

This article shows a methodology which focuses on pysical spaces computational virtualization. Virtualization is understood as projects in which a physical space is digitalised in an interactive way. The metodology is shown in details, showing those common problems regarding this kind of project as well as suggesting their possible solutions.

\section{KEY WORDS}

Virtualization, interactivity, modelling, texturing and 3D animation. 


\section{Méthodologie appliquée à la numérisation informatique de l'espace physique.}

\section{RÉSUMÉ}

Cet article présente une méthodologie centrée sur la virtualisation de projets d'espaces physiques. La virtualisation modélise des projets dans lesquels un lieu physique sera digitalisé de manière interactive. La méthodologie est présentée en détail ainsi que les problèmes que posent normalement ce type de projets. Les solutions possibles à ces prolèmes seront exposées.

\section{MoTS-CLÉS:}

Virtualisation, interactivité, modélisation, animation 3D. 


\section{Metodologia para virtualização computacional de espaços físicos}

\section{RESUMO}

Este artigo apresenta uma metodología enfocada em projetos de virtualização de espaços físicos. O termo virtualização faz referência a projetos onde digitaliza-se de maneira interativa um lugar físico. A metodologia é exposta em detalhe assim como alguns problemas que geralmente surgem neste tipo de projetos, e suas possíveis soluções.

\section{Palavras-chave:}

Virtualização, Interatividade, Modelagem, Textura e Animação 3D. 


\section{Introducción}

I problema tratado, es llamado aquí virtualización de espacios físicos y consiste en crear un modelo computacional virtual interactivo de un espacio físico dado, en tres dimensiones. Esto quiere decir que los lugares físicos pueden ser visitados de manera virtual, por medio de un conjunto de herramientas ya sean de Software o de Hardware. La principal motivación para su realización es que aún existe una alta restricción física para visitar lugares que algunas personas pueden tener necesidad de acceder, pero que por alguna razón no les es posible hacerlo.

Sobre este tema existe una gran cantidad de literatura. Por ejemplo, se han considerado las visitas a museos de manera virtual (Pollefeys, Van Gool, Akkermans, De Becker, y Demuynck, 2001) o a un lugar prácticamente inaccesible en el espacio, como la Luna o Marte (Noor, 2010). El número de aplicaciones existentes de este tipo de tecnología, tanto a nivel empresarial como a nivel académico, es inmenso; desde visitas cobradas a lugares virtuales a los que no es posible tener acceso (como museos, universidades y sitios de interés, entre muchos otros), hasta aplicaciones que ayudan a visualizar un determinado espacio para su compra (como ocurre en el sector de los bienes raíces), solo por dar dos ejemplos.

Este artículo, presenta un modelo propuesto luego de recoger varias experiencias de este tipo de virtualización dentro del trabajo desarrollado en el grupo de investigación en videojuegos, de la Universidad EAN (Villaquirán y otros, 2013). El objetivo principal de este trabajo, es la creación de un aplicativo que permita visitar de manera virtual un edificio de las instalaciones de la Universidad, llamado internamente: Edificio Nogal.

Se muestra de manera gráfica el proceso de virtualización aquí discutido (figura 1): a la izquierda se tiene una foto del edificio por la cara sur. Por medio de un proceso de medición, diseño, digitalización y programación se pueden virtualizar interactivamente las características del espacio, para llegar al modelo interactivo de la foto de la derecha. En esta imagen por ejemplo, se pueden apreciar algunas de las características del edificio: consta de siete pisos, es de color verde, es rectangular y con algunas 
ventanas que sobresalen. El modelo virtualizado de la derecha, conserva algunas de estas características.

Figura 1. Representación gráfica del proceso de virtualización.
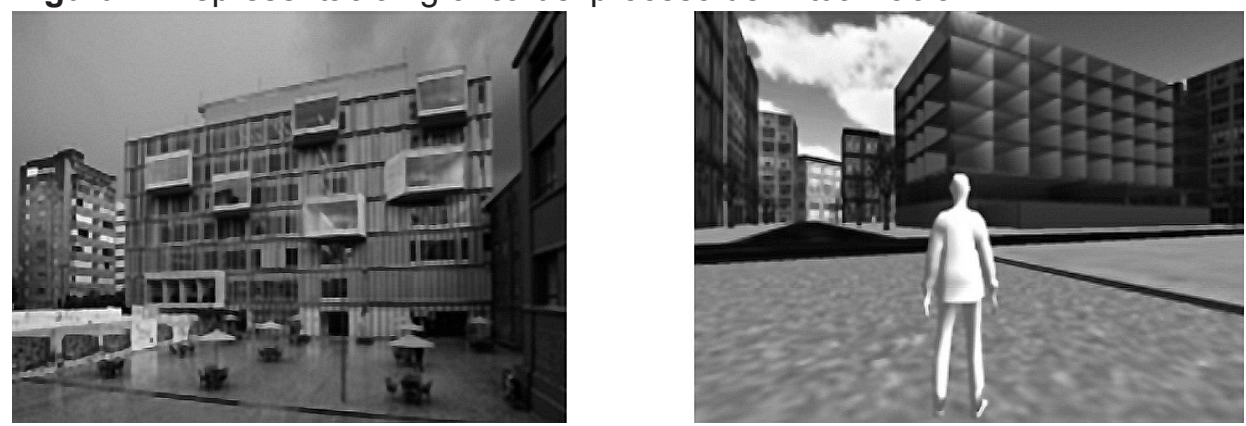

Fuente. Elaborado por el autor.

Este artículo está estructurado de la siguiente manera: en la primera sección se explica la metodología propuesta; en la segunda, se habla de algunos momentos críticos del proceso de virtualización en los que se debe tener especial cuidado. Luego, en los resultados se explican las características del prototipo desarrollado; posteriormente se mencionan los aspectos del trabajo futuro y las diferentes posibilidades que se desprenden de este trabajo. Finalmente, se presentan las conclusiones del artículo. 


\section{Metodología}

T a metodología que se planteó y usó experimentalmente y

la cual condujo al éxito del proyecto, consta de cinco fases. Adicionalmente, se definió una fase de reconocimiento previo del equipo, denominada fase 0 , la cual es importante debido a que en el desarrollo de este tipo de proyectos es necesario utilizar competencias en diferentes tipos de tareas como: edición de imágenes, composición y creación de archivos musicales, creación y edición de modelos en 3D.

Otras metodologías, como las propuestas por Sherwood y Rout (1998) o Sturman y Fabry (1997), se enfocan en el mejoramiento de la calidad, en especial de los recursos multimedia. La diferencia con otras publicaciones similares radica en que en este artículo se propone una metodología en la adquisición de datos (Assets) para desarrollar modelos interactivos. También se tuvieron en cuenta metodologías de tipo general como la RUP (Jacobson, Booch, y Rumbaugh, 1999) y cascada o desarrollo por prototipos (Pressman, 2010).

En la metodología propuesta (figura 2), se observa el proceso metodológico de manera secuencial. El proceso general comienza en la fase cero, donde se recoge información del proyecto y se prepara al equipo de trabajo en relación con el ambiente y con una capacitación o nivelación previa. En la fase 1 se considera el proceso de análisis de requerimientos y evaluación de herramientas, por lo que es necesario hacer estas dos actividades en paralelo. 
Figura 2. Modelo gráfico de la metodología usada.

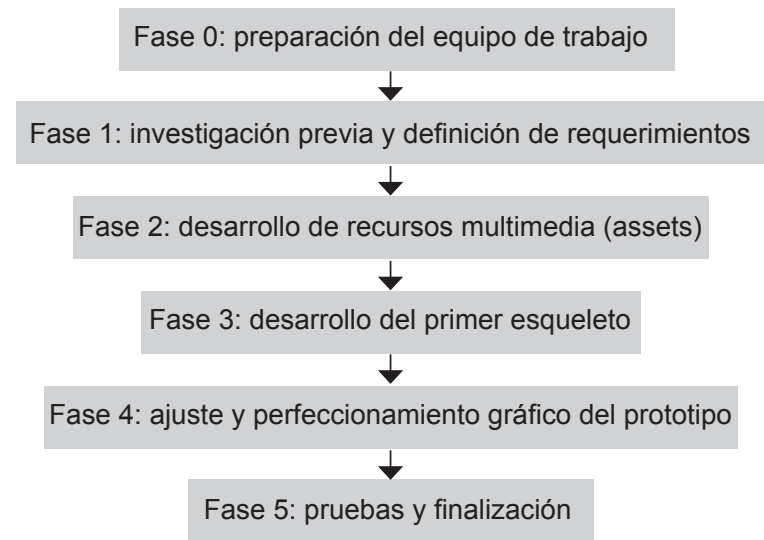

Fuente. Elaborado por el autor.

En la fase 2, empieza el proceso de desarrollo de los diferentes elementos multimedia, siempre teniendo en cuenta cuales son las herramientas que se usarán para darle la parte dinámica o interactiva. En la fase 3 se plantea el desarrollo de un esqueleto de aplicación inicial que luego en la fase 4 se mejorará agregando detalles y elementos multimedia extra. Finalmente, en la última fase se hacen diferentes pruebas y se realiza una parte de entrega o despliegue. A continuación se describe de manera más detallada cada una de las cinco fases propuestas.

\subsection{Fase Cero: conocimiento del grupo de trabajo}

A pesar de no estar considerada dentro de la metodología planteada como el primer paso, esta fase es de vital importancia para el proyecto. La razón para no estar incluida en la secuencia de fases como las demás, es que desde cierto punto de vista es una fase opcional pues en un proyecto distinto las capacidades del equipo pueden ser conocidas de antemano.

Conocer las diferentes capacidades del equipo es de vital importancia en este tipo de proyectos, ya que cada integrante puede colaborar de diferentes maneras en el proceso de desarrollo de este tipo de aplicaciones, dado que la cantidad de tareas a realizar es bastante grande: edición 
de imágenes, sonido, medición de distancias, programación, evaluación, creación de elementos 3D, búsqueda de recursos, entre muchas otras. Por ello es tan importante entender cuáles son las fortalezas del equipo, para poder maximizar el trabajo a realizar.

\subsection{Fase 1: investigación previa y definición de requerimientos}

El objetivo de la fase de investigación previa y análisis o definición de requerimientos, es seleccionar un conjunto de herramientas para empezar a trabajar. Estos aspectos están directamente relacionados, debido al gran cambio que hay en las herramientas de edición de gráficos y modelos 3D, motores de juegos, recursos disponibles, entre muchos otros factores.

\subsection{Fase 2: desarrollo de recursos multimedia (Assets)}

Una vez se hayan definido las herramientas a utilizar, es necesario empezar a crear todos los elementos gráficos y multimediales (Assets) que se decidan crear. Esto se debe hacer con una planeación clara que sea acorde a los objetivos y requerimientos definidos en la etapa previa. También es importante hacer una primera organización de los roles en el equipo de trabajo, donde se distribuya el trabajo de acuerdo a las fortalezas de cada uno de los integrantes.

Otro factor a tener en cuenta, es el tipo de archivos, escalas, resoluciones y estándares con los que se crearán estos Assets y en general, todos los elementos multimedia. No tener en cuenta la escala en la que se crearán los modelos, por ejemplo, es una falla frecuente que puede generar una unificación incompleta al momento de ser mezcladas. De la misma manera, el equivocar un formato se puede llegar a dañar gran parte del trabajo realizado por otros integrantes. 


\subsection{Fase 3: desarrollo del primer prototipo}

Cuando se tengan los elementos gráficos suficientes de acuerdo a la planeación inicial, es posible empezar el desarrollo de un primer prototipo que comience a reflejar la principal funcionalidad que se pretende implementar. Siempre se tiene que tener en cuenta que el prototipo a realizar debe poderse modificar de manera simple, ya que a este esqueleto se le añadirán la mayoría de elementos multimedia que están siendo creados en paralelo. Aunque en esta fase se integran parte de los principales elementos gráficos (Assets gráficos) a la herramienta/motor elegida/o para el desarrollo del proyecto, otros pueden ser creados posteriormente.

También es posible que en esta fase haya una reorganización de los roles en el equipo de trabajo; de nuevo, teniendo en cuenta las fortalezas de cada integrante. En este caso, se deben escoger los integrantes con capacidad de programación y abstracción superiores.

\subsection{Fase 4: ajuste y perfeccionamiento del prototipo}

Una vez terminada la funcionalidad más importante del prototipo, es posible que ya se tenga la primera versión funcional. Sin embargo, en todo proyecto de este tipo es necesaria una fase donde se pruebe exhaustivamente la funcionalidad relacionada con los elementos gráficos actuales. Además, es necesario hacer un análisis del estado visual del prototipo que conlleve su perfeccionamiento, hasta lograr un estado agradable para el usuario. El prototipo inicial podría ser agradable para los desarrolladores, pero siempre es necesaria una segunda opinión crítica que agregue valor a la percepción del usuario final, con el fin de que su experiencia sea lo más agradable posible.

\subsection{Fase 5: pruebas y despliegue del prototipo}

Como fase final es necesario realizar un nuevo conjunto de pruebas donde se asegure una ejecución completa, sin errores y sin inconvenientes mayores. Aunque una buena metodología y buenas prácticas aseguran 
un decremento en la cantidad de errores, esto no garantiza un producto totalmente libre de imperfecciones debido a que la interacción con elementos es muy compleja. Por lo tanto, es necesario realizar este conjunto de pruebas donde se encuentren errores tanto en los Assets, como en la programación lógica.

También es necesario probar el producto final en dispositivos reales, ya sean consolas, computadores de escritorio o dispositivos móviles. Mucha de la programación o desarrollo puede hacerse en emuladores desde computadores personales, pero siempre es necesario verificar y detectar posibles errores que se encuentran a la hora de hacer un despliegue formal del aplicativo. 


\section{Obstáculos frecuentes en el proceso de virtualización}

continuación se detallan diferentes problemas que generalmente se presentan durante la realización de proyectos de este tipo. En particular, se discuten la importancia y los efectos que tiene la selección del Software de animación, relacionada con las herramientas de modelado 3D usadas; la selección del equipo de trabajo, teniendo en cuenta las fortalezas individuales para la creación de elementos gráficos; la integración de elementos con las diferentes herramientas; y los detalles de implementación de la funcionalidad en general.

\subsection{Elección del Software de animación y modelado 3D}

Esta selección se detalla aquí como un factor crítico de este tipo de proyectos. Cuando se analizan las herramientas disponibles, se encuentra una gran variedad de Software como 3D Studio Max, Maya y Blender, entre muchos otros. De estas herramientas, hay algunas que entran en la categoría de Software libre mientras que otras, como 3D Studio Max o Maya, son de tipo propietario, donde su uso implica incurrir en gastos de licencias o uso de versiones de prueba. Esto puede hacer que un proyecto se retrase debido a los trámites necesarios para acceder a una licencia por parte de la entidad financiadora. Mientras que otras herramientas como Blender, permiten realizar lo mismo y además son de Software libre bajo licencia GPL.

Algo importante, es que al no ser las herramientas más populares en la industria, el proceso de producción de Assets gráficos es un poco diferente al utilizado por las empresas de desarrollo de aplicaciones multimedia similares. Sin embargo, este tipo de herramientas cuenta con una documentación y comunidad de usuarios bastante activa a nivel mundial, lo que permite que el proceso de aprendizaje y establecimiento de un Pipeline de producción se encuentre acorde a las necesidades del proyecto. 


\subsection{Creación de Assets gráficos y asignación de roles de trabajo inicial}

Se puede presentar el caso en el que el perfil profesional de los miembros del grupo de investigación sea de ingenieros o estudiantes de ingeniería y no de diseñadores gráficos o modeladores/animadores 3D. En estos casos, el proceso de creación de Assets gráficos, y el proceso de entrenamiento en el uso de las herramientas para la creación de los mismos, es bastante lento en comparación a la realización por parte de profesionales o personas afines a esta área.

Una solución a este inconveniente, es dividir el grupo en roles enfocados a la creación del arte, desde los inicios del proceso de desarrollo del prototipo y cuando se tenga un avance suficiente se puede empezar a asignar roles en tareas afines a ingeniería de Software. De esta manera, se logra generar el suficiente material gráfico en un rango de tiempo aceptable, a pesar de la falta de personas afines al diseño gráfico dentro del grupo.

Generalmente, las tareas de creación de elementos gráficos (Assets) se dividen en las siguientes categorías:

- Creación de los elementos estáticos del ambiente virtual, que no necesariamente son una representación 1:1 de los elementos presentes en el entorno físico, como por ejemplo edificios cercanos, aceras, árboles, cielos, montañas, etc.

- Creación de elementos estáticos navegables y representaciones de elementos físicos existentes, como por ejemplo los propios edificios de la Universidad, el interior de los mismos con base en fotografías, planos e información suministrada por la Universidad.

- Creación de los avatares o personajes con los cuales el usuario puede navegar a través del entorno virtual. Esto incluye modelado y animaciones (caminar, estar de pie, etc.).

También se pueden presentar inconvenientes al trabajar de forma colaborativa sobre un mismo Asset. En muchos casos, los avances de un integrante son sobre escritos por otro integrante durante el proceso de creación. Sin embargo, aunque existe la posibilidad de utilizar soluciones para trabajo colaborativo y control de versiones (repositorios como 
GitHub, Subversion, CVS), el problema radica en el tipo de archivos que se manejan en las herramientas, puesto que son archivos binarios que no pueden ser unificados sin intervención directa de los autores, a diferencia de los archivos de código fuente que son textos planos.

Para solucionar esto sin incurrir en gastos extra con soluciones especializadas para el manejo de archivos binarios, se puede establecer un conjunto de prácticas internas que permitan al equipo de trabajo conocer el estado actual de un determinado archivo binario. También se aconseja establecer responsables por elemento gráfico, de tal manera que esta persona indique a los diferentes integrantes las posibles correcciones para hacer en cada elemento.

\subsection{Integración de Assets y uso del motor/herramienta elegida}

Otro inconveniente que puede surgir es la integración de los elementos gráficos creados por las herramientas de modelamiento 3D, con la herramienta de desarrollo que permite crear la interacción. Entre los principales inconvenientes se encuentra que la escala de los objetos como edificios, paredes o escaleras puede variar entre sí. La falta de un estándar definido para la creación de los elementos puede hacer que elementos visuales como los colores de las paredes y los pisos varíen un poco y se deban modificar para ser incluidas de manera adecuada. El problema es de fácil solución, si al inicio del proyecto se definen estándares que estén de acuerdo a las características identificadas como necesarias en el motor en el cual se implementará la parte interactiva.

Si no se establecen estándares, es posible que se llegue a un punto en el que los recursos desarrollados difieran con respecto a medidas y colores. En este caso, una de las estrategias que se puede usar es repartir nuevamente los roles del equipo, de manera que un grupo de personas se enfoque en el desarrollo de Software de elementos para dar un Look and Feel acorde a lo esperado (como la interacción del usuario con el mundo, cámaras virtuales o efectos gráficos desarrollados en el motor); mientras que las personas restantes pueden enfocarse tanto en la finalización y mejora de los elementos gráficos existentes como en 
la creación de nuevos objetos como el avatar que representa al usuario.

Es necesario que el proceso de creación de animaciones se realice de forma sincronizada con la programación, debido a que existen ciertas reglas impuestas por parte del conocimiento técnico hacia el desarrollo del arte, que deben cumplirse para lograr los efectos deseados.

\subsection{Desarrollo de la interacción}

Otro punto clave en el desarrollo de este tipo de proyectos, es el desarrollo de la interacción del usuario con el mundo. Generalmente, para indicarle al usuario que está dentro del mundo virtual, se crea un avatar genérico o personaje, con el cual este puede recorrer el entorno. Para lograr la funcionalidad de navegación virtual en el mundo, es necesario realizar la programación de las acciones de traslación y rotación de objetos dentro del mundo virtual, así como el uso de un sistema de detección de colisiones por medio de un motor de física especializado, proveído por el motor de juego.

Esto hace que en el prototipo se vean reflejadas ciertas restricciones físicas, como que el personaje o avatar no atraviese paredes o tenga la capacidad de subir pisos en los edificios. En el caso de no usar un personaje, de igual manera es posible añadir ciertas restricciones sobre el mundo; por ejemplo, que la cámara no atraviese las paredes o que tenga una interacción similar a la de una persona caminando.

En cualquier caso, este proceso se debe realizar de la mano con los responsables del área de arte, debido a que es necesario incluir ciertas características dentro de algunos de los elementos visuales. Por ejemplo, si elementos como las escaleras son demasiado altas, el avatar no podría subirlas o bajarlas. Para minimizar este tiempo de desarrollo con los creadores de arte, es importante implementar una estrategia que permita crear elementos de navegación temporales o de detección de colisiones con los elementos gráficos parciales (no definitivos) que simulen la interacción, de tal forma que si algo no funciona se pueda corregir a tiempo, sin la necesidad incluir los objetos complejos. 


\section{Conclusion}

T a metodología aquí planteada, ha sido probada y mejorada a través de diversos proyectos de este tipo. Trabajos como los de Villaquirán y otros (2013), donde esta es usada se muestran que es posible desarrollar proyectos de virtualización con una alta efectividad, aún en condiciones de carencia de recursos. En el trabajo reportado, por ejemplo, se presenta un caso de falta experiencia del equipo de trabajo. También se muestra si bien es necesario un trabajo adicional en la parte gráfica, se puede crear una experiencia similar a la de otros productos del mismo estilo, usando la metodología propuesta, aún sin contar con expertos o personas afines al diseño gráfico.

No obstante, es importante hacer énfasis en que el desarrollo de este tipo de aplicaciones requiere un trabajo considerable de arte (modelado, texturizado y animación 3D). Esto indica que en trabajos similares es recomendable contar con una persona experta en el área de arte digital dentro del equipo, para agilizar el proceso de desarrollo; así como de expertos en la generación de elementos Graphic User Interface (GUI).

Otra área que se debe abarcar en trabajos futuros, es el desarrollo de audio. Esta es una parte importante para la inmersión en la virtualización $3 \mathrm{D}$, en la que también lo ideal es contar con la ayuda o colaboración de un integrante experto, tanto en creación musical y de sonido como en archivos de sonido a nivel informático.

La ventaja en el uso de las herramientas y la metodología de desarrollo en el proyecto, permite expandirlo a través de extensiones en forma bastante sencilla para el equipo de investigación, así como también hace posible incluir equipos multidisciplinarios para enriquecer y mejorar la experiencia del usuario. 


\section{Referencias bibliográficas}

Jacobson, I., Booch, G., \& Rumbaugh, J. (1999). The Unified Software Development Process. New York: Addison-Wesley Professional.

Noor, A. (2010). Potential of virtual worlds for remote space exploration. Advances in Engineering Software, Volume 41, Issue 4.

Pollefeys, M., Van Gool, L., Akkermans, I., De Becker, D., \& Demuynck, K. (2001). A guided tour to virtual Sagalassos. Proceedings of the 2001 conference on Virtual reality, archeology, and cultural heritage. Glyfada, Greece.

Pressman, R. (2010). Software Engineering: A Practitioner's Approach. Boston: McGraw Hill.

Sherwood, C., \& Rout, T. (1998). A Structured Methodology For Multimedia Product And Systems Development. Proceedings of the 15th Annual Conference of the Australasian Society for Computers in Learning in Tertiary Education(ASCILITE). Australia.

Sturman, J., \& Fabry, D. (1997). Teaching Students the Techniques and Tricks to Create Successful Multimedia Projects (The Easy Way). ACM SIGGRAPH Computer Graphics, 80 - 81.

Villaquirán, D., Julián Mora, D., Arizabaleta, et. al. (2013). EAN Virtual: experiencia de virtualización $3 D$ de un edificio en un prototipo dinámico. Revista Virtu@Imente, 1 (2) Bogotá: Universidad EAN 70-80. 